Cahiers $d u$ MONDE RUSSE

\section{Cahiers du monde russe}

Russie - Empire russe - Union soviétique et États indépendants

$59 / 4 \mid 2018$

Varia

\title{
Karol Modzelewski, Nous avons fait galoper l'histoire
}

Confessions d'un cavalier usé

Catherine Gousseff

\section{(2) OpenEdition}

1 Journals

Édition électronique

URL : https://journals.openedition.org/monderusse/10982

DOI : 10.4000/monderusse.10982

ISSN : $1777-5388$

Éditeur

Éditions de l'EHESS

\section{Édition imprimée}

Date de publication : 1 octobre 2018

Pagination : 735-738

ISBN : 978-2-7132-2747-9

ISSN : $1252-6576$

Référence électronique

Catherine Gousseff, « Karol Modzelewski, Nous avons fait galoper I'histoire », Cahiers du monde russe [En ligne], 59/4 | 2018, mis en ligne le 01 octobre 2018, consulté le 06 janvier 2022. URL : http://

journals.openedition.org/monderusse/10982 ; DOI : https://doi.org/10.4000/monderusse.10982

(c) École des hautes études en sciences sociales 


\section{Karol MODZELEWSKI}

\section{Nous avons fait galoper l'histoire}

\section{Confessions d'un cavalier usé}

(ouvrage traduit du polonais par Elżbieta Salamaka),

Paris : Éditions de la Maison des sciences de l'homme, 2018, 541 p.

Nous devons à Karol Modzelewski d'avoir baptisé « Solidarité » le plus grand mouvement social de l'Europe communiste, une intuition au diapason de son combat historique pour un socialisme démocratique. S'il se dépeint aujourd'hui comme un cavalier « usé ", l'usure ne se réfère qu'à l'anti-solidarité des temps présents. L'autobiographie réflexive de ce grand militant de l'opposition dans la Pologne de la répulique populaire (et après) retrace les étapes d'une lutte collective dans le récit de soi, où le « je » est situé avec une justesse saisissante, à travers l'environnement social, les époques, une manière sûre d'y dépeindre sa propre subjectivité, manifestant dans une grande plénitude l'historien qu'est aussi Karol Modzelewski. Ces Confessions ne sont pas seulement les mémoires d'un acteur majeur de l'histoire contemporaine polonaise, elles sont aussi un grand livre d'histoire qui bénéficie du recul analytique d'un intellectuel hors pair.

Parmi les multiples fils que suit ce livre, entre parcours politique, trajectoire d'historien, portrait d'une société dans la diversité des milieux croisés - celui de l'intelligentsia certes, mais aussi celui du monde ouvrier et de l'univers carcéral, Karol Modzelewski l'ayant fréquenté pendant plus de huit ans - on choisira de suivre d'abord le fil le plus personnel de l'auteur, tel qu'il se présente dans une autobiographie qu'il aborde en particulier à travers la question de son identité et de son lien à la nation.

Le « je » de ce livre présente en effet une trajectoire personnelle exceptionnelle. Né en 1937 à Moscou d'un père officier, bientôt arrêté et d'une mère juive russe, 
K. Modzelewski a vécu la guerre dans une maison d'enfants près de Gor'kij, qu'il a quittée imprégné de l'atmosphère ambiante, en patriote radical, envisageant le monde sous la forme de deux blocs, les Russes et leurs ennemis, les Allemands. Russe, l'enfant est convaincu de l'être à son retour à Moscou, malgré le changement de décor puisqu'il se retrouve désormais à l'ambassade polonaise où son père adoptif, Zygmunt Modzelewski, assume la plus haute charge. Étrange destin pour ce dernier qui, à l'instar d'un certain nombre de communistes polonais, est passé par l'épreuve des répressions staliniennes avant d'être appelé à reconstruire la Pologne d'après-guerre, en l'occurrence pour Zygmunt Modzelewski au poste de ministre des Affaires étrangères. Retracer dès lors, depuis Varsovie, comment le petit soldat russe dans l'âme est devenu un Polonais qui revendique son attachement à la nation, revient à évoquer un processus. Ce processus se réfère certes à un apprivoisement linguistique et culturel, mais il s'inscrit surtout dans un cheminement politique où se révèle, eu égard à la précocité des convictions et leur centralité dans la personnalité de Karol Modzelewski, l'impact décisif des bouleversements introduits avec la mort de Stalin. La loyauté de l'adolescent au camp soviétique avait été à peu près à cette époque ébranlée par la découverte des « excès » du stalinisme faite, surtout, à l'occasion des révélations tardives de ses deux pères, biologique et d'adoption, sur l'univers concentrationnaire et la pratique de la torture, technique ordinaire pour forcer l'aveu. C'est ensuite à la lumière du Rapport de Hruščev (qui, fait spécifique au sein du camp socialiste, fut publié et largement diffusé dans le pays), de l'automne polonais de 1956 et de son premier engagement militant que l'auteur réalise « qu'il n'était plus russe, mais polonais ». Cette mutation identitaire qui correspond à un choix politique n'est pas qu'un constat. Elle se traduit dans un comportement moral très engagé qui inspire plusieurs réflexions sur la nation. Par exemple, lorsqu'en évoquant, en 1968, la participation des militaires polonais à l'intervention des armées du Pacte de Varsovie en Tchécoslovaquie, Karol Modzelewski dit sa honte, en la présentant comme un sentiment élevé. Car, poursuit-il, « la honte (...) est un signe infaillible de lien national ». Contrairement à la culture européenne qui ne croit pas en la responsabilité collective et ne juge les actes condamnables qu'à travers leurs auteurs, « quiconque revendique vraiment l'appartenance à la communauté nationale éprouve nécessairement de la honte pour les actes infâmes perpétrés par ses participants et en son nom ». On pourrait à cela ajouter que si les Européens de l'Ouest se distinguent en effet de leurs voisins plus à l'Est, ils épousent volontiers leur conception du lien national lorsqu'il s'agit de les juger... De ce lien qualifié de central dans ses expériences, Karol Modzelewski l'éclaire de multiples manières au fil des pages, depuis le contexte de "l'empire soviétique » et la réactivation des traditions politiques de la Pologne, entre romantisme et réalisme pragmatique, jusqu'à un certain respect manifesté par le pouvoir à la figure de l'intellectuel de la nation, quand bien même rebelle, en l'occurrence ici du doctorant médiéviste autorisé derrière les verrous à consulter son directeur de thèse, à rédiger ses travaux... Malgré la centralité des confrontations vécues, l'attention portée aux signes, même ténus, de complicité entre « eux et nous » participe aussi à l'intelligibilité de ce lien. 
Suivre à présent le parcours militant de Karol Modzelewski dans ces pages conduit à retracer, pour ceux qui la connaissent mal, l'histoire politique de la Pologne populaire depuis le dégel. 1956 est une année clé pour l'étudiant en histoire de l'université de Varsovie, alors en pleine ébullition, qui rencontre Jacek Kuron avec lequel il formera dans les décennies à venir un duo infernal pour le pouvoir en place. Elle l'est aussi par sa première approche du milieu ouvrier, qu'il aborde en représentant de l'Union de la jeunesse polonaise (ZMP, équivalent des Komsomols) auprès de l'usine FSO de Varsovie en lutte pour la création de conseils ouvriers et l'autogestion des entreprises. La rétrospective de cette mobilisation est l'occasion pour l'auteur de dresser un magnifique portrait de Leszek Goździk, le « leader charismatique » de l'Octobre polonais qui délaissera la scène politique après la normalisation conduite par Gomulka.

L'expérience initiatrice de 1956 marque le début d'une réflexion critique sur le système, nourrie en partie de l'intérieur, au sein du Parti auquel Karol Modzelewski adhère en 1957 et dont il est expulsé en 1964, à quelques mois de la diffusion de la Lettre ouverte au Parti Ouvrier Unifié polonais (POUP, PC polonais), fruit d'un échange collectif et qu'il cosigne avec Jacek Kuron. Reconnue comme l'acte fondateur de la naissance de l'opposition, cette Lettre ouverte dresse un véritable réquisitoire sur la nature du pouvoir de l'État dit socialiste. Pensée dans les canons du marxisme, l'analyse critique du système conduit à dresser un véritable programme de restauration du pouvoir ouvrier par l'autogestion des entreprises et la création de syndicats professionnels indépendants... Quinze ans plus tard, cette revendication s'incarnera dans l'issue de la grève des chantiers navals de Gdansk et la naissance de Solidarité, une expérience courte, étouffée en moins de deux ans, mais qui aura l'impact que l'on sait et dans laquelle le duo jouera avec Wałęsa et bien d'autres un rôle de leaders... Entre-temps, la Lettre ouverte, diffusée sous la forme de quatorze exemplaires ronéotés, fait le tour du pays, est traduite dans plusieurs langues. Elle coûte à leurs auteurs un premier procès et une incarcération de deux ans. Libérés en 1967, Kuron et Modzelewski se retrouvent à nouveau derrière les barreaux après la mobilisation étudiante de mars 1968 dont ils sont accusés d'avoir été, avec Adam Michnik notamment, les instigateurs. Fait intéressant, lors de ce procès, certains dirigeants firent pression pour que les rebelles soient condamnés en tant que membres de la IVe Internationale trotskiste, et ce afin de renforcer leur position vis-à-vis de Moscou où l'accusation de trotskisme restait même après la mort de Stalin l'épouvantail suprême. Par ce biais, comme dans bien d'autres passages, l'auteur rentre dans l'épaisseur des luttes internes qui se sont jouées au sein du Parti, éclairant la crise politique de 1968 à travers les résonances qu'elle eut jusque dans le procès.

Les années de prison et l'épreuve de l'isolement, mettent à distance le militant mais cette distance est aussi, à divers moments de la trajectoire de Karol Modzelewski, un choix assumé pour poursuivre son travail d'historien médiéviste. Bénéficiaire d'un poste de l'Académie des sciences après sa seconde détention, il reprend ses travaux, désormais à Wrocław qui sera sa ville d'élection. Si le public non polonophone n'a découvert que tardivement son œuvre (son grand ouvrage L'Europe 
des Barbares : Germains et Slaves face aux héritiers de Rome est paru en français en 2006), il est en revanche tôt identifié dans la communauté internationale des spécialistes, bénéficie de plusieurs invitations à l'étranger, en Italie notamment, dès le début des années 1960 et plus tard en France, à l'EHESS. Malgré sa passion pour les époques reculées, l'histoire en train de se faire réimpose ses priorités à la fin des années 1970. S'il n'a pas directement participé à l'aventure du Comité de défense des ouvriers (KOR) fondé en 1976, il « replonge » dans l'activité politique lors de la naissance du syndicat Solidarité, officiellement au titre de représentant élu des entreprises de la région de Wrocław à partir de septembre 1980.

C'est autant en acteur qu'en historien que ce plus gros chapitre de l'histoire est abordé, dans l'exposition méticuleuse des enjeux et des conflits qui ont ponctué le développement de cette aventure collective extraordinaire, aussi bien dans le face-à-face avec le pouvoir qu'entre les membres, socialement et politiquement très divers, de la direction de Solidarité. Cette rétrospective est aussi faite d'arrêts sur images, dépeignant l'atmosphère dense, euphorique et néanmoins tendue de ces mois d'intenses échanges et promesses, l'incarnant à travers de nombreux portraits et situations, n'épargnant pas les critiques à l'égard de certains, à commencer par le tribun Wałęsa, tout en les argumentant avec élégance et profond respect. De cette " première » ou « grande » Solidarité, comme il la nomme tour à tour, l'auteur souligne à quel point elle répondait à ses espérances, ou, plus précisément « au mythe de (sa) jeunesse » étant « un mouvement égalitaire, communautaire et socialiste ». Une assertion concrétisée de multiples exemples, tel celui des chauffeurs de tramway de Wrocław qui, tout en gagnant quatre fois plus que la majorité des ouvriers de la région, rejoignirent sans hésiter le mouvement de grève pour l'augmentation des salaires de ces derniers.

Du coup d'État du général Jaruzelski et de la proclamation de l'État de guerre - la reprise en main du pays par l'armée ayant entraîné pour l'auteur une nouvelle incarcération de plusieurs années - Karol Modzelewski consacre une longue analyse pour en montrer les incidences profondes et irréversibles dans la destruction du mouvement. C'est à l'aune de l'élan brisé, de l'espoir enterré qu'il aborde la fin et l'après de la Pologne socialiste, le tournant néolibéral radical dont il fut l'un des rares à s'opposer, divergeant cette fois même de Jacek Kuron, son plus proche compagnon dans la lutte d'une vie pour l'avènement d'une société plus juste. Karol Modzelewski n'a pas fait le choix, lui, de devenir un politicien dans la nouvelle Pologne. Son « examen d'expériences » s'achève en 2013, portant l'empreinte d'un espoir déçu, mais non vaincu. Dans la tourmente populiste qui a depuis envahi la sphère politique polonaise, et au-delà, il demeure une voix critique, affranchie et vigilante dans ses appels « à ne pas réveiller la bête immonde».

\section{Catherine Gousseff}

CERCEC, CNRS, EHESS, PSL

Piast-PAN, Varsovie 